\title{
Design and Motion Planning of a Mechanical Snake
}

\author{
Yansong Shan and Yoram Koren, Senior Member, IEEE
}

\begin{abstract}
The paper presents the design and motion planning for a mechanical snake robot that was built at the University of Michigan. The structure of the robot enables it to move without wheels. It is constructed of a series of articulated links, each one with a motor and linear solenoid. Although each link has only one motor, this structure allows the body configuration to be easily controlled thereby enabling the robot to move in very cluttered environments. The motion planning system provides the robot with a basic motion pattern that can be easily modified for different tasks and environments. The mechanical snake does not avoid obstacles on its way, but rather "accommodates" them by continuing its motion towards the target while in contact with the obstacles. With our design and motion planning, each link has a different number of degrees-of-freedom in each motion stage, providing the robot with great adaptability even during contact with obstacles in a cluttered environment.
\end{abstract}

\section{INTRODUCTION}

A ROBOTIC DEVICE that is able to crawl into places that are too dangerous or too small for people to enter would find many applications in industry. For example, the most recent design of new nuclear power plants contains a reactor vessel with an 8 -in space between its bottom and the floor. The bottom of the vessel must be inspected for leaks. A small, flexible robotic device could perform this inspection. Leaks or other problems in municipal sewer systems or utility tunnels could also be investigated and perhaps repaired by such a robot. In an earthquake or other disaster situation, it is oftentimes too hazardous for humans to enter collapsed structures. A robot that could maneuver through the rubble to look for survivors would be invaluable. The commonly utilized wheeled robots cannot fulfill the task because of their large size. Even if they are designed to be small, they cannot carry the tools needed to do the job. Surveillance tasks in the military are sometimes highly dangerous, exposing soldiers to hostile fire or mine fields. A robot that could unobtrusively enter the area and travel to a target while delivering intelligence data would reduce wartime casualties.

Robotic devices currently in use do not have the ability to carry out the tasks described above-they are either too large, are not able to move freely in obstacle-cluttered environments, or have wide turning radii. In this research, we have developed a prototype robot with special motion and size characteristics that make it ideal for carrying out tasks that require maneuverability in rough terrain or tight spaces.

We began our research by examining standard methods of robot locomotion: legs, wheels, and tracks. These proved to

Manuscript received July 18, 1992; revised December 16, 1992. This work was supported by the National Science Foundation under grant IRI-9112717.

The authors are with Department of Mechanical Engineering and Applied Mechanics, University of Michigan, Ann Arbor, MI 48109

IEEE Log Number 9209631. be unsatisfactory for meeting the requirements of our robot, so we decided to look for a new mode of robot locomotion. We turned our attention to the study of an animal that has the size and movement characteristics we wanted to create in our device-the snake. We set out to create a robot that could mimic the motion of a snake and would be able to move quickly and unobtrusively in cluttered or tight environments.

Since the snake robot is used in obstacle cluttered environments or very tight spaces, we had to develop a new motion control method called obstacle accommodation to control its motion. This method, in contrast to existing obstacle avoidance motion control methods, does not avoid physical contact between robot and obstacles. Instead, it allows the contact, but controls the motion to avoid damage to the robot. This method is particularly useful for robots that work in natural environments where to totally avoid obstacles is difficult or impossible. It is also useful for robots in the environments where avoiding obstacles will prevent the robot from performing its task. For example, suppose a robot is used in nuclear power plant to perform inspection of an area that is obstructed by objects. Common obstacle avoidance algorithms will not allow the robot to inspect the area. In this situation, allowing contact between the obstacles and the robot enables the robot to successfully inspect the area. The motion controller for the snake robot obstacle accommodation will be discussed in a different paper [14], but the design issues of the robot concerned with contact with obstacles (such as the mobility of links in contact with obstacles) will be discussed in this paper.

In this paper, we present our study of the snake robot including 1) body structure and general motion pattern, 2) motion planning toward an arbitrary target, and 3) mobility after contact with an obstacle.

\section{Body Structure and General Motion Pattern}

The main goal in designing a snake-type robot is to imitate the body configuration and hence the motion control abilities of a real snake. Our approach to designing the snake robot proceeded through three stages. First, we examined the biomechanics of snake motion. From that study, we learned how a snake provides itself with driving force and how it controls its body configuration to achieve motion. Second, we designed a body structure that can mimic a snake's motion without using legs or wheels. Third, we designed the basic motion pattern for the robot. In this section, we examine each of these stages.

\section{A. The Biomechanics of Snake Motion}

Snake propulsion can be classified into four patterns [8]: lateral undulation, rectilinear locomotion, sidewinding, and concertina progression. 

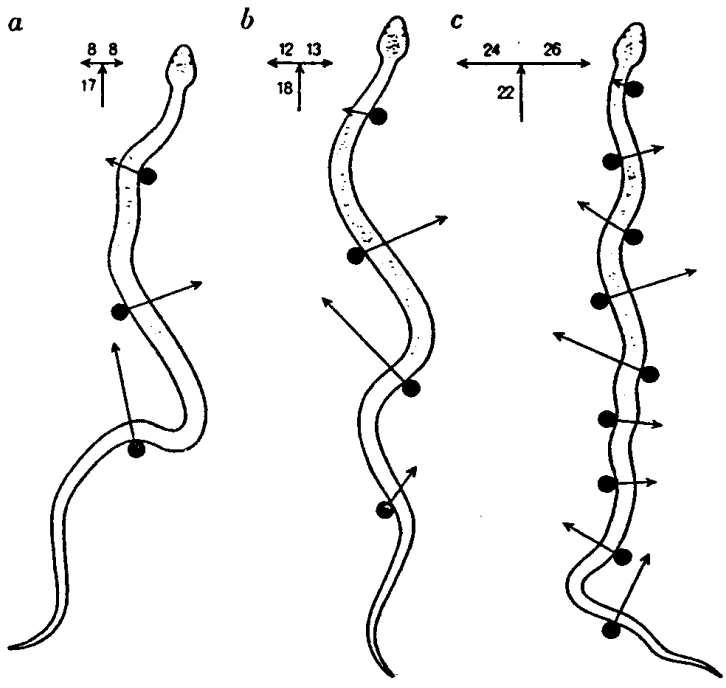

Fig. 1. Lateral undulation motion of snakes.

Lateral undulation is the most common method of movement for snakes. As the name indicates, this movement combines a sideways motion with a body undulation. The snake first moves sideways against objects such as rocks, plants or debris, enabling it to grip the ground at places along its body. It then uses its rib muscles to push off from each contact point starting from the head and moving backward, creating the forward movement. Lateral undulation is the most efficient pattern because it uses normal contact force to drive the snake forward (see Fig. 1 [8]), whereas the other three motion patterns mainly use friction force. The normal and tangential parts of the contact forces have been calculated by Hirose[9] revealing that the normal part of the contact force can be quite large.

Rectilinear locomotion is the second basic motion pattern of snakes. This movement differs from lateral undulation in two respects: it involves the application of force from contact points that are on the bottom of the body instead of on the side, and it is effective only if friction is established between the snake's "skin" and the ground. This motion enables a snake to advance in a straight lines as it stalks prey or crosses a flat surface. In order to move in this mode, the snake fixes several points along the bottom of its body and moves the part of its body between them. (These points fixed on the ground are called static points). The propelling force driving the snake is primarily the friction force between the snake and the ground. Interested readers should refer to [8] for further details.

The third pattern of snake motion is called sidewinding. This pattern enables the snake to employ friction forces without sacrificing speed. Although a sidewinding snake swings through loops that are similar to lateral undulations, the force-transmission pattern is more like that of the concertina sequence (below). The force for the initial acceleration of the front part of the snake and for maintaining the velocity of the moving parts must be transmitted by the friction of the snake's belly. This motion pattern is typically used in sandy or slippery environments.

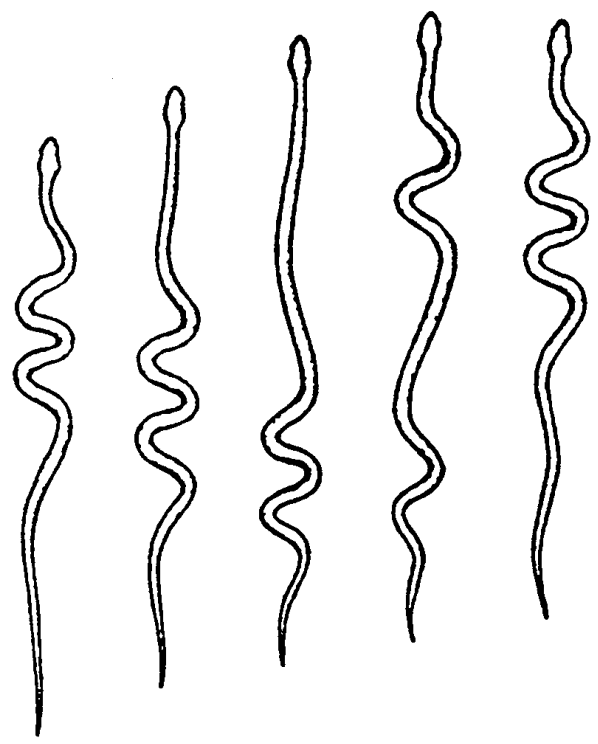

Fig. 2. Concertina motion of snakes.

Concertina motion makes it possible for snakes that do not have the muscle and bone structures necessary for rectilinear progression to still use static friction forces in locomotion. This pattern enables a snake to move in a narrow channel. The snake draws itself into a S-shaped curve similar to the posture assumed in lateral undulation and sets the curved portion of its body in static contact with the ground. The motion begins when the head, the neck, and the forward part of the body are extended by forces from the rear part of the body. The forces are the reaction force transmitted to the ground in the zone that remains in stationary contact with the ground. These static zones play the role of generating the propelling force. Fig. 2 shows the typical shape of the snake body in concertina motion. In this motion, the front end of the snake moves forward a short distance and establishes a new zone of stationary contact in which horizontal forces are exerted against the ground. Then the rear end of the snake's body is pulled forward. Loosely speaking, in concertina motion the snake uses frictional forces to push or pull itself. One of the main reasons that the concertina motion has a lower motion efficiency is because it uses friction force as the driving force. However, an advantage of this motion is that the body shape in this pattern can be quite narrow.

By comparing design results from different approaches, we found that emulating the concertina motion pattern of real snakes with modifications appears to be a good solution in designing the basic motion pattern for the mechanical snake.

\section{B. Body Structure}

Some studies on the design of very flexible articulation structures have been conducted by several researchers. Hirose began investigating the importance of the structure of an articulated body from the point of view of snake biomechanics [9] and designed a few multi-link mobile robots [10]. Taylor 


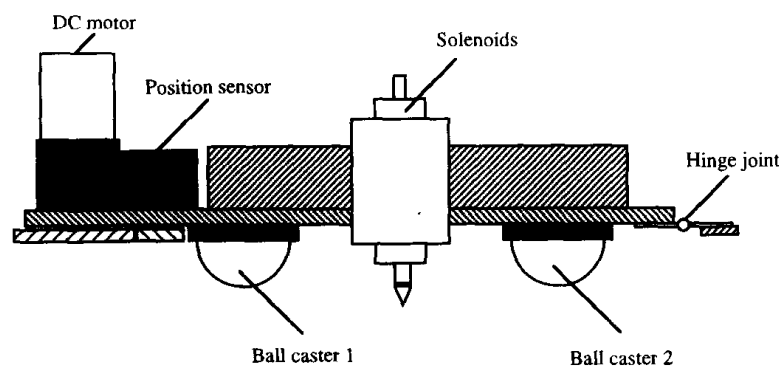

Fig. 3. Link structure.

considered the design of manipulators that consist of connected disks [16] . Baldur and Blach designed an inflatable central cylinder to enhance the flexibility of manipulators [2]. Clement and Inigo designed a snake-like manipulator in which all of the joints are driven by a single prime mover and the snakelike motion ensures its obstacle avoidance [7] . Chirikjian and Burdick studied kinematics and obstacle avoidance algorithm for hyper-redundant robots [5], [6]. Except for Hirose's design, all the structures are for robotic arms with fixed bases. Hirose's articulated mobile robots can move on a surface. They are primary wheel driven, and their body posture while in motion is relatively difficult to control when compared to robot manipulators. His latest version of the articulated mobile robot, KR-I, has many actuators for each individual articulation [10].

In our design, the mechanical snake consists of links. The links are connected through active joints that allow motion in the horizontal plan. To achieve the best posture-control ability, our structure is designed with each joint directly controlled by a DC motor (see Fig. 3). Each DC motor is used to control the angle between one link and the next. A position sensor is used for feedback control of the joint angle.

The challenge is to achieve forward motion of the snake robot when each of its links can move only in the lateral direction. This is achieved by providing programmable contact points with the ground. It is important that these contact points be provided mechanically so that the snake robot can generate push force whenever it is needed. In our design, linear solenoids with sharp tip pins are used to provide static points with the ground and to provide a large contact force (see Fig. 3). When the solenoids are activated (i.e. the pins are inserted into the ground), relatively large contact forces can be generated at the pintips. Each link contacts the ground either directly through the link surface or through two ball casters that, ideally, have less friction force than the link surface. It should be noted that this basic structure can be varied to suit a particular environment or task. For example, a modification of the solenoid design could cause the tip pins to be pushed deeper into the ground in sandy environments where shallow contact would not provide the necessary force.

An additional hinge-type joint between each link allows the snake robot to compensate for sharp irregularities on the ground's surface. The motion of each hinge joint, or the vertical degree-of-freedom of each link, is not controlled by the robot controller, but instead are passively controlled by the geometry of the ground. All the links of the robot are designed

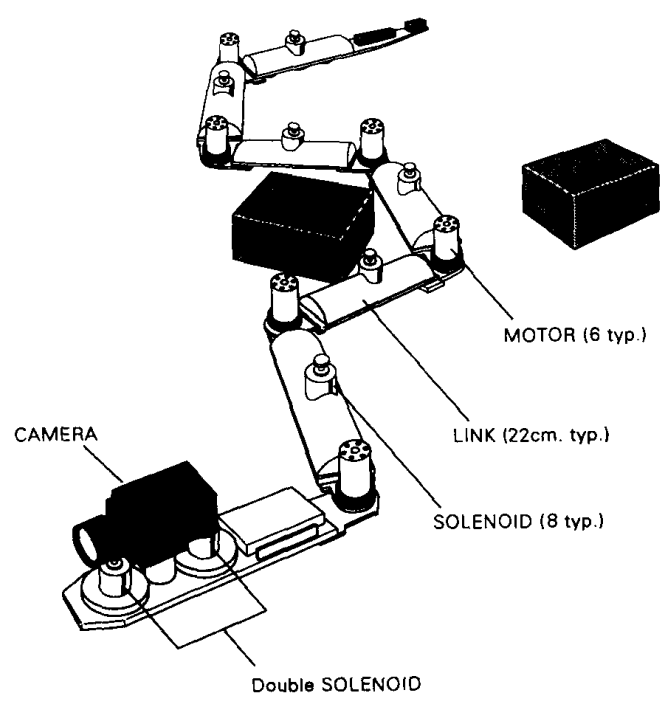

Fig. 4. The mechnanical snake, MS-1.

in the same way except the tail and the head links, which are not connected at one of their ends. In addition, the head link has two solenoids. We have designed two mechanical snakes. Fig. 4 shows the structure our first prototype, MS-1 (Michigan Snake 1). As shown in the figure, MS-1 has a total of 7 links, six controlled joints, six hinge joints, and eight solenoids. Each link of MS-1 are supported by two ball casters that reduce the friction between the snake and the ground. (Our second snake robot, MS-2 (Michigan Snake 2), has no ball casters, the link surfaces contact ground directly)

When the solenoids in a link is activated, i.e. it contacts (inserts into) the ground, a larger force can be generated for that link compared to that of other links, which are solely supported by the ball casters or body surfaces. The motors of the body control the joint angles, and reaction forces at the solenoids vary in magnitude and direction so as to keep the links fixed in position. The controlled forces from the pintips of the solenoids are the driving forces moving the robot forward.

For purposes of discussion in later sections of the paper, we have assigned coordinate systems to each of the snake joints. The tail link is defined as link 1 , the next one is link 2 , and so forth to the head link that is defined as link 7 . The joint coordinate $O_{i}$ is attached to the link with $X_{i}$ axis along the vector from joint $i-1$ to joint $i, Z_{i}$ axis being vertical to the plane that the snake lays, and $Y_{i}$ being determined by the right-hand rule. Fig. 5 shows the coordinate assignments. In the figure, $\boldsymbol{p}_{w}$ is the vector from the world coordinate origin to the first joint, and $\phi_{0}$ is the orientation of $\mathrm{O}_{1}$ (the first joint coordinate system) relative to the world coordinate.

The snake structure and its motion pattern (developed below) are for operating in cluttered environments where easy control of the body posture is necessary. The robot has lower motion efficiency compared to wheeled mobile robots. However, it is important to note that wheels can be added to the snake robot so that it can move faster in environments that are simple and free of obstacles. In these environments, the 


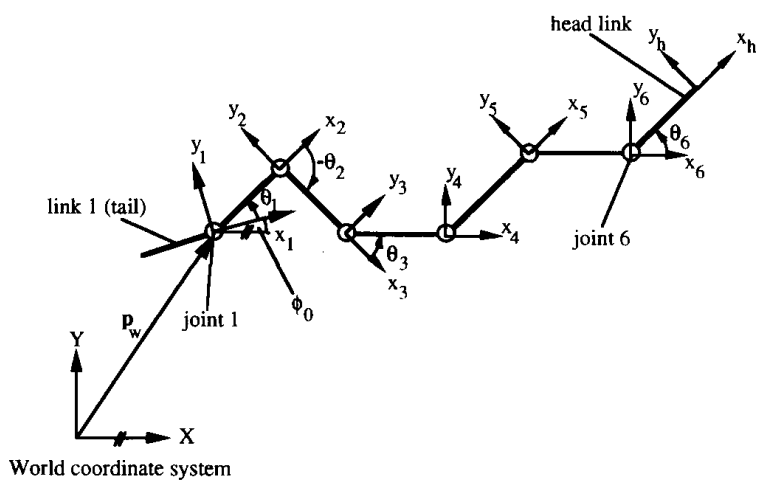

Fig. 5. The coordinate system of the mechanical snake.

mobility of the linkage for controlling the body posture is not needed. An example of wheeled multi-link mobile robot can be found in [10]. In this paper, we only consider the motion control and planning issues for the snake robot that is driven by the joint motors and the solenoids.

\section{Basic Motion Pattern}

Although the motion strategy and motion pattern of the mechanical snake will need to be quite complex due to the variety of work environments and motion tasks it will be required to perform, we have designed a basic concertina motion pattern leaving it to the real-time motion controller to make changes or modifications to suit different work environments and tasks. This is similar to what happens in human locomotion. Although the motion pattern for human movement changes greatly in different environments or for different motions, speeds, and directions, the basic pattern of swinging the two legs backward and forward remains the main characteristic of all human motion actions.

The concertina motion pattern has a very regular and smooth motion sequence. It should be noted that our design eliminates one of the disadvantages of this motion pattern for real snakes. In real snakes, low-efficiency friction force from underneath the body is the driving force. In the mechanical snake, the linear solenoids provide enough contact force to make it the driving force.

Designing the basic motion pattern means determining a particular timing function for the solenoids and the joint motion. Let $\boldsymbol{T}=\left[t_{1}, t_{2} t_{3}, t_{4}, t_{5}, t_{6}, t_{7}, t_{8}\right]^{T}$ represent the timing function of the solenoids, where $t_{i}(i=1, . .8)$ can be either 0 or 1 . Here 0 represents a solenoid that is nonactive and 1 represents one that is active. For instance, $\boldsymbol{T}=[0,0,0,0,0,0,1,1]^{T}$ represents the state in which solenoids 1 to 6 are nonactive and solenoids 7 and 8 are activated, i.e. they insert into the ground, generating a large driving force that propels the snake robot forward. Also let $\Theta(t)\left(\Theta(t)=\left[\theta_{1}(t), \theta_{2}(t), \ldots, \theta_{6}(t)\right]^{T}\right)$ be the joint motion. The basic motion pattern is defined by selecting a particular $\boldsymbol{T}$ and $\Theta(t)$.

The basic motion pattern for the snake robot is created through a series of identical motion cycles. Each motion cycle
TABLE I

Motion Sequence of the Basic Motion Pattern

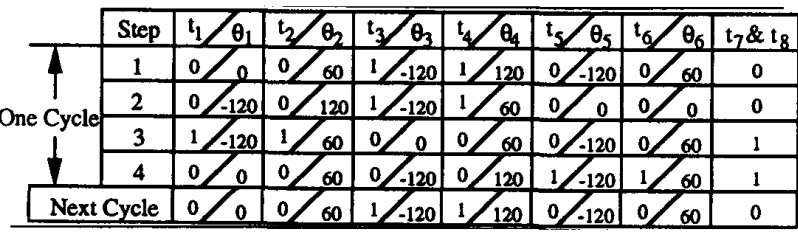

Note: 1) "1" means the solenoid of the link is active and " 0 " means it is released; 2) Joint angles are in degrees.

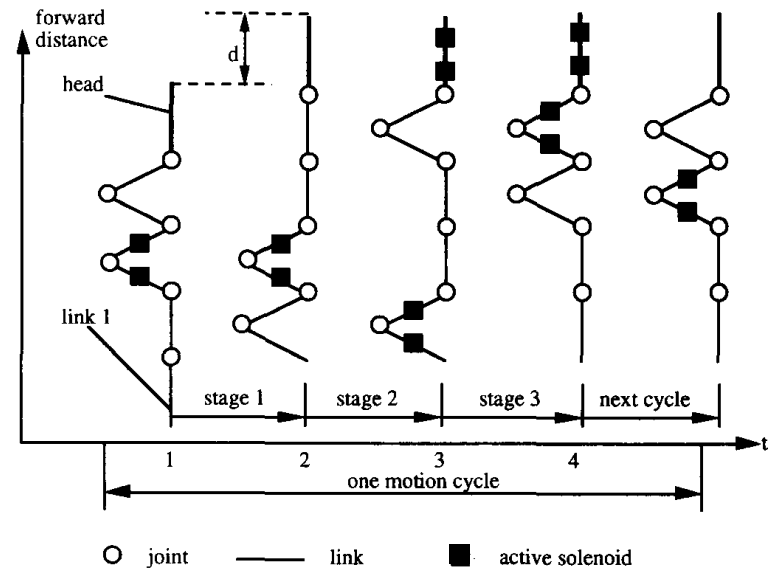

Fig. 6. The basic motion pattern.

has three stages: the first stage pushes the head part of the robot (links 5, 6, and the head) forward; the second stage pulls the middle part of the body forward (links $3,4,5$, and 6), and the last stage pulls the tail part (links 1,2,3, and 4) up. One cycle of the basic motion pattern is given in Table I and depicted in Fig. 6. (Note the resemblance between Fig. 2 and Fig. 6.) As a result of this sequence, the head advances a distance $d$ in every motion cycle. Note that the snake does not move between step 4 and step 1 of the next cycle; only the active solenoids are changed. In Table I, the angles are measured in a local joint coordinate system. The values, $60^{\circ}$ and $120^{\circ}$, are two typical values for the joint angles in experiments. These values are subject to change in real application depends on the environments, motion direction, and motion tasks.

The snake design and the basic motion pattern enable the motion controller to control the motion of the snake in a way similar to controlling robot manipulators. In the first stage, links 5,6 , and 7 form an open linkage, and links 1 and 2 form another open linkage. In the second motion stage, links $2,3,4,5$, and 6 form a closed linkage. In the third stage, links $1,2,3$, and 4 form an open linkage. It is clear that in terms of the body posture and link position control, the manipulator type of mechanism is much easier than the wheel driven articulated mobile robots. This is especially important in cluttered environments.

The lateral undulation is a very efficient motion pattern for real snakes; however, this motion is possible only if there are lateral objects that exert force on the snakes. For the 


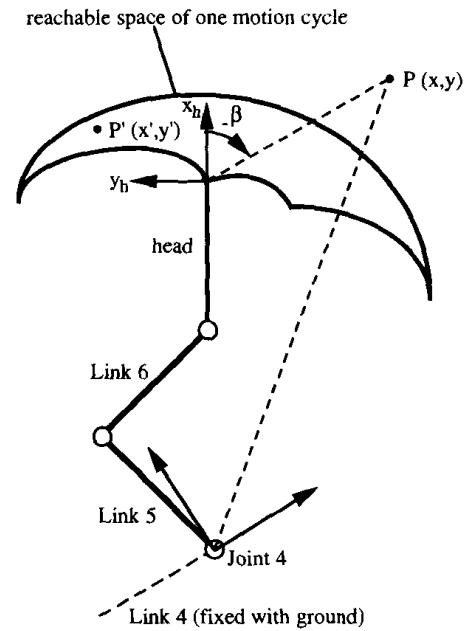

Fig. 7. Target position inside and outside reachable space.

mechanical snake, the solenoids are used to provide the force for the motion. Overall, the mechanical snake moves forward by mimicking the concertina motion with the driving forces from the solenoids underneath the body.

As mentioned earlier in the section, the basic motion pattern can be changed for different motion tasks and environments. In the next section, we discuss how to modify the basic motion pattern so the robot can move toward an arbitrarily assigned target that is not straight ahead of it.

\section{Motion TOWARD AN ARBITRARY TARGET}

In this section, we discuss a modification of the basic motion pattern of the mechanical snake robot that allows it to reach an arbitrarily assigned target in Cartesian space that is not straight ahead. Since each motion cycle has three motion stages, we discuss the modification of each motion stage.

\section{A. Motion of the First Stage}

In the first motion stage, links 3 and 4 are stationary, and links 5, 6, and 7 (the head) form a standard three DOF planar linkage. The task of standard robot motion planning can usually be stated as the following: move the joints so that the end effector will reach a target in a specified orientation. With the snake robot, we cannot state the motion-planning problem as simply as this, since the target is not usually inside the current reachable space. We define the current reachable space as the set of points in Cartesian space that can be reached by the snake head after the current motion cycle. Fig. 7 shows a reachable envelope of the first motion stage (it is the same as the robot work envelope). Since the head link is stationary after the first motion stage, the reachable envelope of the first stage is the same as the current reachable space of the motion cycle.

If the target position, $P^{\prime}$, is inside the current reachable space, the motion of the three links is the same as of a planar three-link manipulator, and the angle change during the motion stage can be calculated using inverse kinematics. However, in this paper, we are more concerned about the motion planning for reaching a target position outside the current reachable space, as $P$.

Suppose at time $t_{0}$ the snake is at the stage just before its first motion stage, and the target, $P$, is outside the current reachable space, as shown in Fig. 7. (In the figure, $\beta$ and $d$ represent the target position in terms of the head-coordinate system.) Since the joint coordinate system $\mathrm{O}_{4}$ is fixed with the world coordinate system, the target is also constant in $\mathrm{O}_{4}$. The target position in terms of the joint coordinate system $O_{4}$ is

$$
\begin{aligned}
& x_{p 4}=\left[L_{5} C_{4}+L_{6} C_{45}+L_{7} C_{456}+d \cos \beta\right]_{t=t_{0}} \\
& y_{p 4}=\left[L_{5} S_{4}+L_{6} S_{45}+L_{7} S_{456}+d \sin \beta\right]_{t=t_{0}}
\end{aligned}
$$

where $S_{4}=\sin \theta_{4}$ and $S_{45}=\sin \left(\theta_{4}+\theta_{5}\right)$, etc., and $\theta_{4}, \theta_{5}$, as well as $\theta_{6}$ are positions of joints 4,5 , and 6 respectively. Note that $x_{p 4}$ and $y_{p 4}$ are constants during the first motion stage. The distance between the head position and target position can be calculated as

$$
\begin{aligned}
d^{2}= & {\left[L_{5} C_{4}+L_{6} C_{45}+L_{7} C_{456}-x_{p 4}\right]^{2} } \\
& +\left[L_{5} S_{4}+L_{6} S_{45}+L_{7} S_{456}-y_{p 4}\right]^{2}
\end{aligned}
$$

At the end of the stage, the goal is for the distance $d$ to be the minimum value. The values of joint angles 4,5 , and 6 at $t=T_{f 1}\left(T_{f 1}\right.$ is the time when the robot finishes its first motion stage) can be found by solving

$$
\begin{aligned}
\frac{\partial d^{2}}{\partial \theta_{4}}= & {\left[L_{5} S_{4}+L_{6} S_{45}+L_{7} S_{456}-y_{p 4}\right] } \\
& \times\left(L_{5} C_{4}+L_{6} C_{45}+L_{7} C_{456}\right) \\
& -\left[L_{5} C_{4}+L_{6} C_{45}+L_{7} C_{456}-x_{p 4}\right] \\
& \times\left(L_{5} S_{4}+L_{6} S_{45}+L_{7} S_{456}\right) \\
= & 0 \\
\frac{\partial d^{2}}{\partial \theta_{5}}= & {\left[L_{5} S_{4}+L_{6} S_{45}+L_{7} S_{456}-y_{p 4}\right]\left(L_{6} C_{45}+L_{7} C_{456}\right) } \\
& -\left[L_{5} C_{4}+L_{6} C_{45}+L_{7} C_{456}-x_{p 4}\right]\left(L_{6} S_{45}+L_{7} S_{456}\right) \\
= & 0 \\
\frac{\partial d^{2}}{\partial \theta_{6}}= & {\left[L_{5} S_{4}+L_{6} S_{45}+L_{7} S_{456}-y_{p 4}\right] L_{7} C_{456} } \\
& -\left[L_{5} C_{4}+L_{6} C_{45}+L_{7} C_{456}-x_{p 4}\right] L_{7} S_{456} \\
= & 0
\end{aligned}
$$

The solutions to the above three equations are

$$
\theta_{4}=\tan ^{-1}\left(\frac{y_{p 4}}{x_{p 4}}\right), \theta_{5}=0, \text { and } \theta_{6}=0 .
$$

Fig. 8 shows a motion sequence of the robot as it reaches an arbitrary target in its first motion stage.

In Fig. 8, $\alpha$ is the angle between the head link before and after the first stage. During stage 1 , links 3 and 4 remain stationary and joint 3 does not change, i.e. $\theta_{3}\left(T_{f 1}\right)=\theta_{3}\left(t_{0}\right)$, where $t_{0}$ is the time when the snake starts the current motion cycle. Simultaneously with the motion of links 4,5 , and 6 , links 1 and 2 are simply folded as when the robot moves in the basic motion pattern. After the first motion stage, we have $\theta_{1}\left(T_{f l}\right)=\theta_{1}^{0}\left(T_{f l}\right)$ and $\theta_{2}\left(T_{f l}\right)=\theta_{2}^{0}\left(T_{f l}\right) . \theta_{1}^{0}\left(T_{f l}\right)$ and $\theta_{2}^{0}\left(T_{f l}\right)$ are the angles in the basic motion pattern at time 

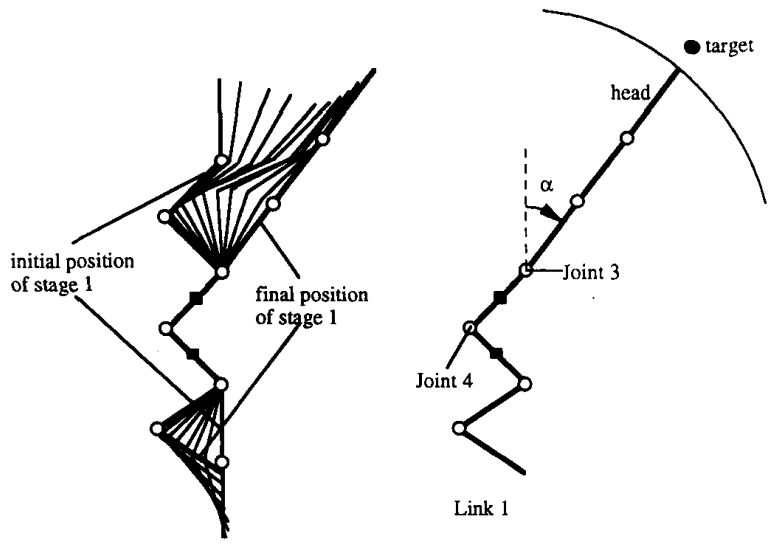

Link 1

Fig. 8. The first motion stage

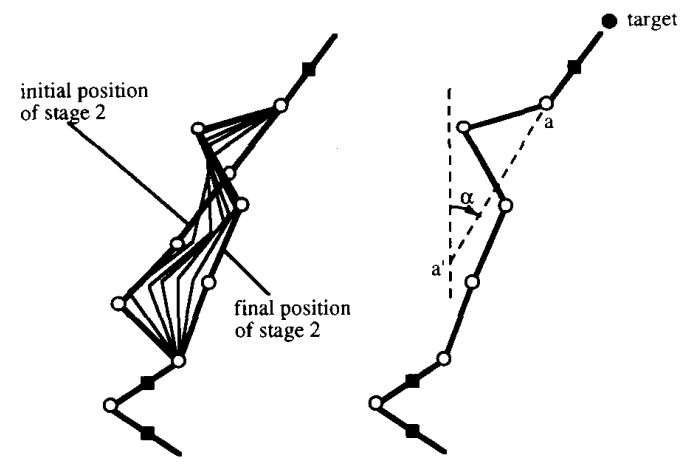

Fig. 9. The second motion stage.

$t=T_{f l}$. In summary, after the first motion stage, the joint positions are

$$
\theta_{1}\left(T_{f 1}\right)=\theta_{1}^{0}\left(T_{f 1}\right), \theta_{2}\left(T_{f 1}\right)=\theta_{2}^{0}\left(T_{f 1}\right), \theta_{3}\left(T_{f 1}\right)=\theta_{3}\left(t_{0}\right),
$$

and

$$
\theta_{4}\left(T_{f 1}\right)=\tan ^{-1}\left(\frac{y_{p 4}}{x_{p 4}}\right), \theta_{5}\left(T_{f 1}\right)=0, \text { and } \theta_{6}\left(T_{f 1}\right)=0 .
$$

\section{B. Motion of the Second Stage}

In the second motion stage, links 1,2 , and 7 are stationary. The snake pulls links 5 and 6 up and, at the same time, extends links 3 and 4. Links 3 to 6 form a closed kinematic chain system with two degrees-of-freedom (DOF). Fig. 9 shows a possible motion sequence in the second motion stage.

In the second motion stage, joint 2 is fixed with the ground, so we can express the motion in terms of joint 2 . After the second motion stage, the distance between joints 4 and 6 is desired be the minimum. Joint 6 is fixed with the ground, and its position in joint coordinate 2 is

$$
\begin{aligned}
& x_{62}=L_{3} C_{2}+L_{4} C_{23}+L_{5} C_{234}+L_{6} C_{2345} \\
& y_{62}=L_{3} S_{2}+L_{4} S_{23}+L_{5} S_{234}+L_{6} S_{2345}
\end{aligned}
$$

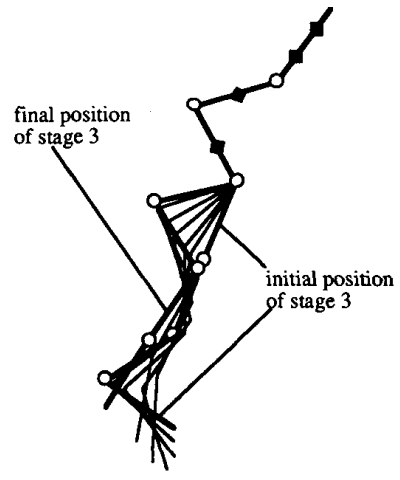

Fig. 10. The third motion stage.

During the motion, the distance between joints 4 and 6 is

$$
d_{64}^{2}=\left(L_{3} C_{2}+L_{4} C_{23}-x_{62}\right)^{2}+\left(L_{3} S_{2}+L_{4} S_{23}-y_{62}\right)^{2} .
$$

The optimum solution can be obtained by solving

$$
\begin{aligned}
\frac{d_{64}^{2}}{\partial \theta_{2}}= & \left(L_{3} S_{2}+L_{4} S_{23}-y_{62}\right)\left(L_{3} C_{2}+L_{4} C_{23}\right) \\
& -\left(L_{3} C_{2}+L_{4} C_{23}-x_{62}\right)\left(L_{3} S_{2}+L_{4} S_{23}\right)=0 \\
\frac{d_{64}^{2}}{\partial \theta_{3}}= & \left(L_{3} S_{2}+L_{4} S_{23}-y_{62}\right) L_{4} C_{23} \\
& -\left(L_{3} C_{2}+L_{4} C_{23}-x_{62}\right) L_{4} S_{23}=0 .
\end{aligned}
$$

From (9), the solutions are

$$
\theta_{2}\left(T_{f 2}\right)=\tan ^{-1}\left(\frac{y_{62}}{x_{62}}\right) \text {, and } \theta_{3}\left(T_{f 2}\right)=0 \text {. }
$$

For a given $\theta_{2}$ and $\theta_{3}$, the distance $d_{64}$ is determined, and we can find the angles for joints 4,5 , and 6 by using cosine law:

$$
\begin{aligned}
& \theta_{4}\left(T_{f 2}\right)=\cos ^{-1}\left(\frac{d_{64}^{2}+L_{5}^{2}-L_{6}^{2}}{2 d_{64} L_{5}}\right) \\
& \theta_{5}\left(T_{f 2}\right)=\cos ^{-1}\left(\frac{L_{5}^{2}+L_{6}^{2}-d_{64}^{2}}{2 L_{5} L_{6}}\right)-\pi \\
& \theta_{6}\left(T_{f 2}\right)=\cos ^{-1}\left(\frac{d_{64}^{2}+L_{6}^{2}-L_{5}^{2}}{2 d_{64} L_{6}}\right)-\theta_{2}\left(T_{f 2}\right)+\Theta_{h 2}
\end{aligned}
$$

where $T_{f 2}$ is the time when the second motion stage is finished and $\Theta_{h 2}$ is the orientation of the head link in terms of the joint coordinate 2 , which is constant during the second motion stage. $d_{64}$ is calculated by (8) with $\theta_{2}$ calculated from (10).

\section{Motion of the Third Stage}

Fig. 10 shows the motion sequence of the third motion stage. In this stage, links 5, 6 and the head link are kept stationary during the motion. The reference point for the motion is set at joint 4 .

Since there are no kinematic restrictions to the linkage formed by links $1,2,3$, and 4 at the end of the third motion cycle, joints $1,2,3$, and 4 are moved back to their initial positions, and the whole body configuration is in the initial 
state for the next motion cycle. Throughout the third motion cycle, joints 5 and 6 are constant. The joint position changes can be summarized as follows:

$$
\begin{aligned}
& \theta_{1}\left(T_{f}\right)=\theta_{1}^{0}\left(T_{f}\right), \theta_{2}\left(T_{f}\right)=\theta_{2}^{0}\left(T_{f}\right), \theta_{3}\left(T_{f}\right)=\theta_{3}^{0}\left(T_{f}\right), \\
& \theta_{4}\left(T_{f}\right)=\theta_{4}^{0}\left(T_{f}\right), \theta_{5}\left(T_{f}\right)=\theta_{5}\left(T_{f 2}\right), \text { and } \theta_{6}\left(T_{f}\right)=\theta_{6}\left(T_{f 2}\right)
\end{aligned}
$$

where $T_{f}$ is the time when the third motion stage is finished (the current motion cycle is finished). Since $\theta_{5}\left(T_{f}\right)$ and $\theta_{6}\left(T_{f}\right)$ are functions of the target position and the previous position of the snake relative to the target, the final positions of links $1,2,3,4$ relative to links 5, 6 , and the head link are functions of the target position relative to the previous position of the snake. After the third motion stage, the robot is ready to start the next motion cycle.

We have run experiments to verify the snake's ability to turn and reach an arbitrarily assigned target. A typical motion sequence for reaching an arbitrary target is shown in Fig. 11.

Our second snake robot prototype, MS-2, is shown in Fig. 12. MS-2 has similar structure with MS-1 except that it has two solenoids on every link and the pitch of its head can be controlled. The ability to control the head pitch enables MS-2 to move over obstructions. Once the head clears an obstruction, the remaining links can push the head over the obstruction. Then the snake robot can proceed using the motion pattern discussed above. Another difference between MS-1 and MS-2 is that each link of MS-2 has no ball casters underneath the link.

\section{Kinematics}

In this section, we present a kinematic analysis of the motion of the mechanical snake robot. We discuss 1 ) kinematic equations for Cartesian positioning and 2) the motion properties of an open linkage chain (motion stage 1 and 3 ) and a closed linkage chain (motion stage 2).

1) Cartesian Positioning: As shown in Fig. 5, any point on the snake robot can be determined in the world coordinate system if $\boldsymbol{p}_{w}, \phi_{0}$, and $\theta_{i}(i=1, \ldots 6)$ are known. The snake is controlled to move from its current position at time $t_{0}$ to a new position at time $T_{f}$ where its head advances a distance $d$ and the head orientation changes by $\gamma$ as shown in Fig. 13. We want to calculate the new $\boldsymbol{p}_{w}$ and $\phi_{0}$.

We can express the target position and orientation of the head link in the world coordinate system as

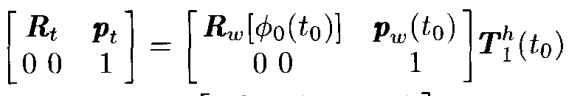

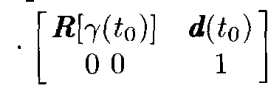

$$
\begin{aligned}
& =\left[\begin{array}{cc}
\boldsymbol{R}_{w}\left[\phi_{0}\left(T_{f}\right)\right] & \boldsymbol{p}_{w}\left(T_{f}\right) \\
00 & 1
\end{array}\right] \boldsymbol{T}_{1}^{h}\left(T_{f}\right)
\end{aligned}
$$

where

$$
R^{2 \times 2}(.)=\left[\begin{array}{rr}
\cos (.) & -\sin (.) \\
\sin (.) & \cos (.)
\end{array}\right]
$$

where $\boldsymbol{R}_{t}^{2 \times 2}$ and $\boldsymbol{p}_{t}$ are the target orientation and position in the world coordinate, $\boldsymbol{R}_{w}$ and $\boldsymbol{p}_{w}$ are the orientation and

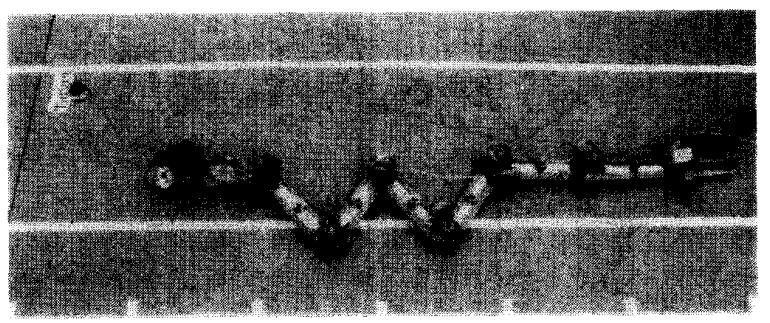

(a)

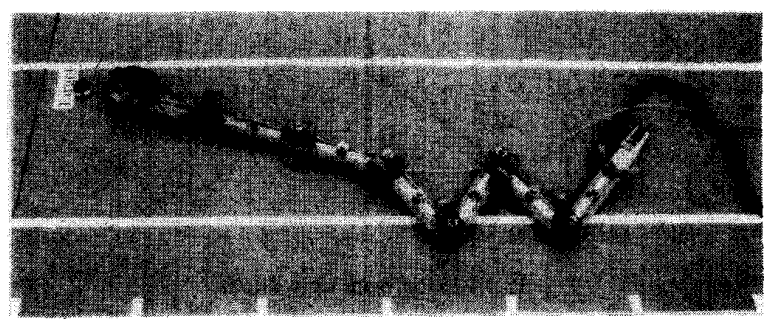

(b)

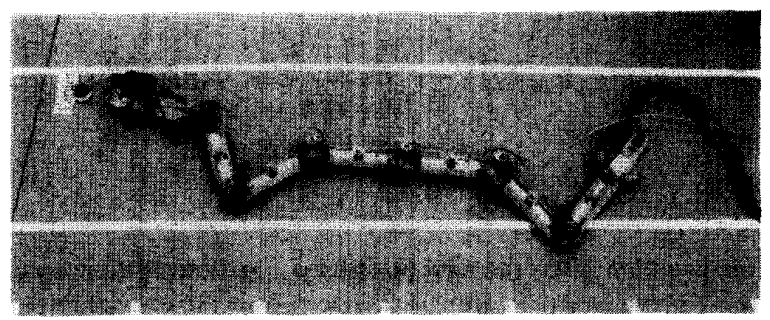

(c)

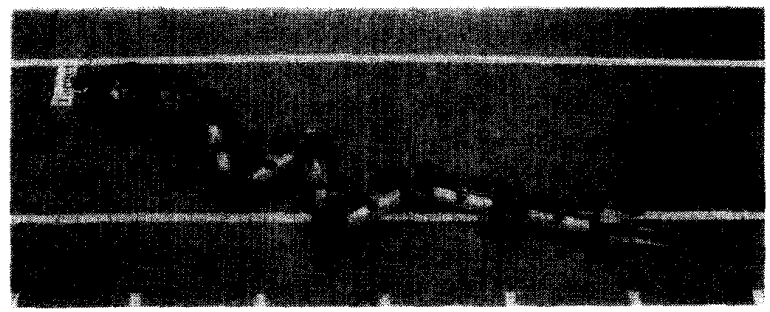

(d)

Fig. 11. Motion towards an arbitrary target. (a) Before motion. (b) After the first stage. (c) After the second stage. (d) After the third stage (the motion cycle completed)

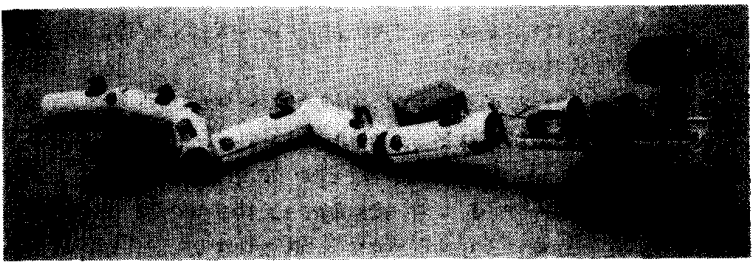

Fig. 12. The mechanical snake MS-2.

position of joint 1 in the world coordinate, and, $\boldsymbol{T}_{1}^{h}(t)$, is the transformation matrix from the joint coordinate 1 to the head coordinate $O_{h}$, and can be determined since it totally depends 

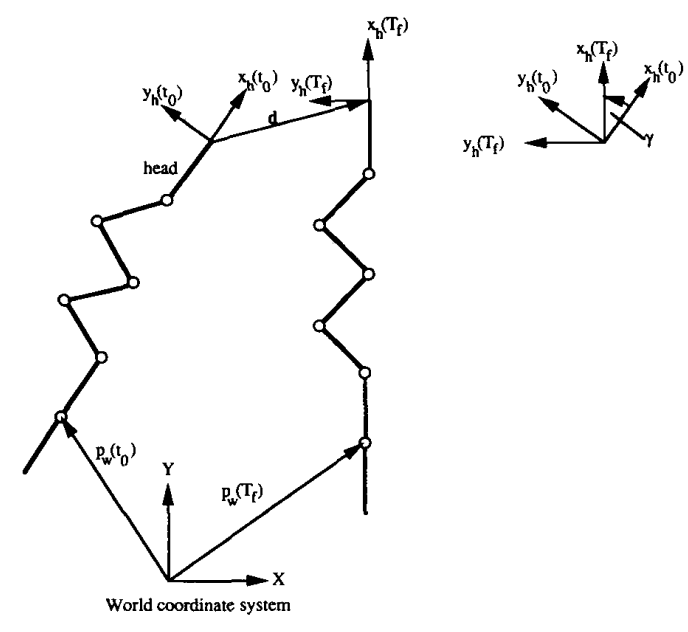

Fig. 13. Motion from time $t$ to $T_{f}$.

on the joint angles that are measurable. From (13) we have

$$
\begin{aligned}
& {\left[\begin{array}{cc}
\boldsymbol{R}_{w}\left[\phi_{0}\left(T_{f}\right)\right] & \boldsymbol{p}_{w}\left(T_{f}\right) \\
00 & 1
\end{array}\right]=\left[\begin{array}{cc}
\boldsymbol{R}_{w}\left[\phi_{0}\left(t_{0}\right)\right] & \boldsymbol{p}_{w}\left(t_{0}\right) \\
00 & 1
\end{array}\right] \boldsymbol{T}_{1}^{h}\left(t_{0}\right)}
\end{aligned}
$$

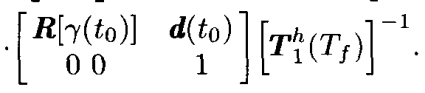

This equation gives the new position $\boldsymbol{p}_{\boldsymbol{w}}$ and orientation $\phi_{0}$ of joint 1 in the world coordinate system that enables to determine the position and orientation of any point on the snake.

2) Open and Closed Linkage Chain: The motion of the snake robot involves open and closed linkage types of motion. The open linkage types of motion are employed in the first and the third motion stages. In the first motion stage, two open linkage chains are formed. The first is formed by links 5,6 , and 7 (head link), and the second is formed by links 1 and 2 (see Fig. 6 and 8). Links 3 and 4 are kept steady by the solenoids. Joint 4 is the origin for the first open linkage, and joint 2 is the origin of the second open linkage. In the third motion stage, links $1,2,3$, and 4 form a open linkage with the origin at joint 4 .

In the first stage, the motion of the three joints $\left(\theta_{4}, \theta_{5}\right.$, and $\theta_{6}$ ) can control the motion of the head link toward a specified position inside the reachable space. Fig. 8 shows the positions of links 5, 6, and 7 before and after the first stage. The kinematics during these two motion stages is the same as that of a robot manipulator.

The closed linkage type of motion is seen in the second motion stage. During the second motion stage, the links in motion are links $3,4,5$, and 6 . The head link (link 7), link 1 , and link 2 are fixed with respect to the world coordinate system. Links $3,4,5$, and 6 form a five-bar closed chain (see Fig. 9).

Joints 2 and 6 are the two fixed ends for the closed loop linkage. This closed linkage has two degrees-of-freedom. There are a total of five joints involved in the second motion stage. However, since there are only two DOF, two of the five joints are selected as the driving joints and the other three are

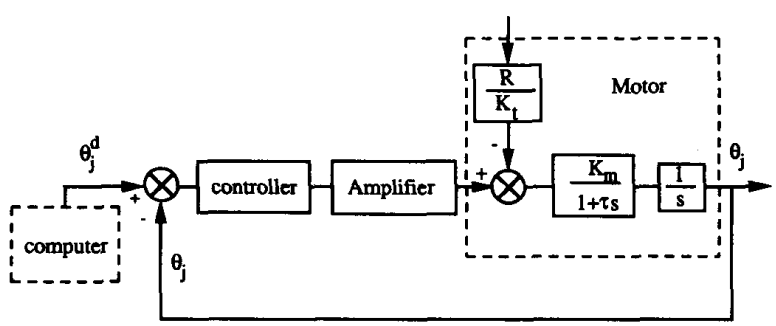

(a)

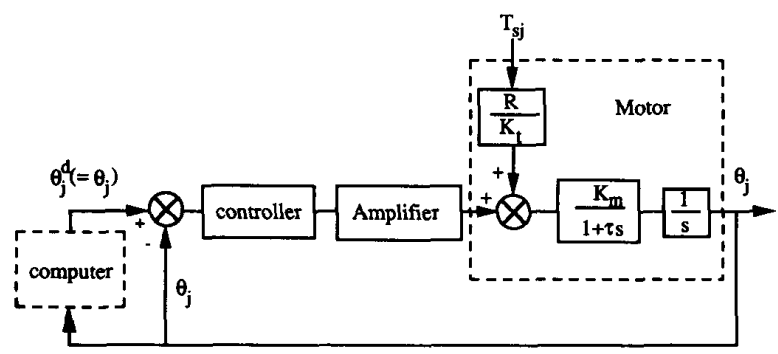

(b)

Fig. 14. Controller structures.

passive joints. In our experiments, joints 2 and 5 are selected as the driving joints.

Fig. 14(a) shows the structure of the controller in the active joints [12]. In the figure, $K_{m}$ is the gain of the motor, $\tau$ is the time constant, $K_{t}$ is the motor torque constant, $R$ is the resistance of armature circuit, and $T_{\mathrm{s}}$ is the external disturbance to each of the active joints.

To make those joints that are not selected as driving joints be passive, namely free rotary joints, we developed a new control method. In our method, the external torque, $T_{s}$, that is now generated by the active joints and operates on the passive joint becomes the input to the controller of the passive joint. As a result, the joint moves in the direction of the external torque. In each sampling period, the computer reads the new value of the joint angle and send it back to the corresponding joint servo-controller as its new input (see Fig. 14b). The position error instantaneously becomes zero. This process continues until every $\theta_{j}$ reaches the value given by the motion planner as the final value of the second motion stage. It should be noted that in the passive joint control loop, the sign of $T_{s}$ is positive since the external torque is the driving force for the motion.

\section{Mobility aFTER CONTACT WITH an OBSTACle}

When working in a cluttered environment, the robot will encounter and contact obstacles that will constrain its motion. As discussed in Section 1, the robot should continue to have mobility after these contacts. In this section, we describe the kinematic feasibility of motion for each stage during contact with obstacles. We focus on providing a basic understanding of the contact effects on snake motion and we omit the mathematical discussion of the kinematics. 


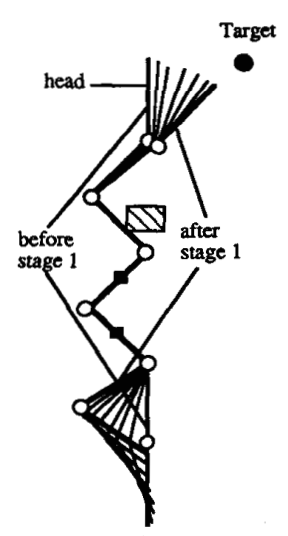

(a)

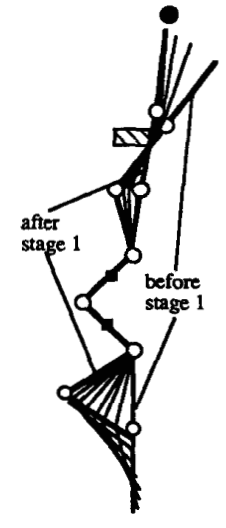

(b)

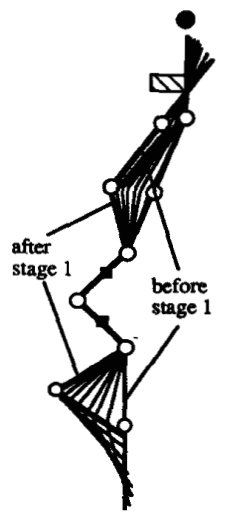

(c)

Fig. 15. An obstable contacts different links in the first motion stage.

\section{A. Motion after Contact}

In this discussion, we assume that the contact between the robot and the obstacle is continuous because once the obstacle is out of contact with the robot, the motion constraints are relaxed and the kinematic properties become the same as when the robot moves in free space.

1) The First Stage: During the first motion stage links 3 and 4 are fixed. Obstacles could contact the snake robot on any moving link, i.e., any link not marked with a diamond in Fig. 15. Suppose that an obstacle contacts link 5, since link 4 is fixed, link 5 has only one degree-of-freedom (DOF) number before the contact. Link 5 does not move further after it first contacts the obstacle, while links 6 and 7 keep moving the head toward the target (see Fig. 15(a)).

Let us consider another case (shown in Fig. 15(b)), where the obstacle contacts link 6 in the first stage. Because the link had two DOF before the contact it now still has one DOF. The link can slide along the obstacle.

Another possible scenario is one in which the head link collides with an obstacle, as shown in Fig. 15(c). The head link originally has three DOF, and the contact reduces that to two DOF. With these two DOF, the head link can be controlled to slide along the obstacle.

Note that when an obstacle collides with links 1 or 2 , the motion properties of these two links are the same as those for the motion of links 5, 6, and 7 from a kinematic point of view, since these two links also form an open linkage chain.

2) The Second Stage: In the second stage, the controller moves links 3, 4, 5, and 6, and keeps the head link and links 1 and 2 stationary with respect to the world coordinate. With these links fixed to the ground, links $3,4,5$, and 6 form a closed loop linkage that has two DOF.

Since links 3 and 6 have zero DOF due to the contact, they cannot move as long as the contact remains. However, since links 4 and 5 have $2 \mathrm{DOF}$, the robot can move further if they encounter an obstacle. Fig. 16(a) shows a possible motion sequence when link 4 encounters an obstacle, and Fig. 16(b) shows the case when the contact is on link 5 .

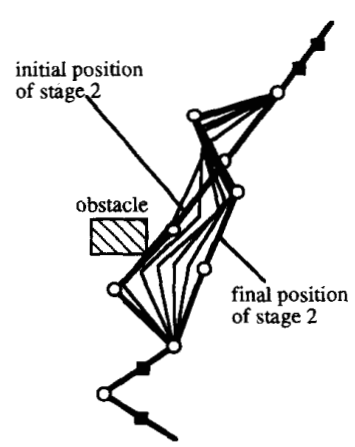

(a)

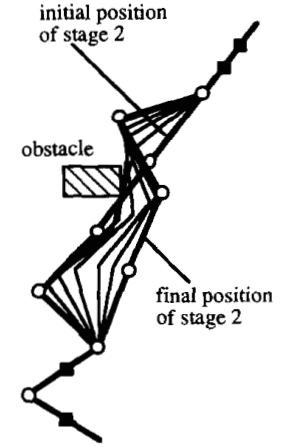

(b)
Fig. 16. An obstable contacts different links in the second motion stage.

TABLE II

DOF of Each Link in Different Motion Cycle

\begin{tabular}{cccc}
\hline DOF & First Stage & Second Stage & Third Stage \\
\hline Link 1 & 2 & 0 & 3 \\
Link 2 & 1 & 0 & 3 \\
Link 3 & 0 & 1 & 2 \\
Link 4 & 0 & 2 & 1 \\
Link 5 & 1 & 2 & 0 \\
Link 6 & 2 & 1 & 0 \\
Link 7 & 3 & 0 & 0 \\
\hline
\end{tabular}

3) The Third Stage: Since the third motion stage also involves open linkage motion, its kinematic properties are the same as those for the first motion stage.

\section{B. Mobility of Each Link}

Examining the snake robot's motion, we find that the number of degrees-of-freedom of each link varies across the three motion stages. Table II tabulates the number of DOF of each link in different motion stages for the cases in which the robot does not contact any obstacle.

In the table, 0 means that the corresponding link has zero DOF; it cannot move relative to the world coordinate (i.e., the ground). As an example, the number of DOF of link 6 is 2 in the first motion stage but 1 in the second motion stage.

From the table, we can see that each link has more than 1 DOF in at least one of the motion stages. This property enables the robot to slide along an obstacle in at least one motion stage no matter which link encounters the obstacle. For instance, if link 5 encounters an obstacle in the first motion stage, that link stays put, since the number of DOF of the link decreases to zero due to the contact. However, in the second motion stage, the same link has 1 DOF even with the contact and can slide along the obstacle.

The different kinematic properties in different motion stages are important because if a link is blocked in one of the motion stages, the controller can still move the link in another motion stage.

In this study, we have assumed that the contact forces between the robot and obstacles can be controlled so that the robot can move without damage while touching obstacles. This assumption is justified by the results of our studies of obstacle 
accommodating controllers [13], [15]. We have developed two types of obstacle accommodation controllers: one type uses tactile sensors while the other does not-using only joint torque sensors instead. Our results show that the controller without tactile sensors accommodates obstacles well in most the situations except when obstacles contact the robot at certain positions. In these situations, the controller needs to know the exact contact position; therefore, a small number of tactile sensors are recommended at these positions. The controller design for the mechanical snake will be presented in a separate paper [14].

\section{CONCLUSION}

This paper presents a study of the design and motion control of a mechanical snake developed at the University of Michigan. The design is unique in that it does not use wheels to drive the robot. It uses a motion that emulates the movement of a real snake. The design provides good flexibility for control of the body posture, since it can control the angle of each joint. We demonstrate the motion with a mechanical snake prototype. The experimental results show that our design has several advantages. It is able to easily turn in any direction, and its body configuration can be controlled even when the snake encounters obstacles. One of the unique kinematic properties of the snake robot is that each link has a different number of degrees-of-freedom in different motion stages, which enriches the mobility of the robot allowing it to move in cluttered environments even when it contacts obstacles.

\section{ACKNOWLEDGMENT}

The authors also wish to thank Mr. Lynn Buege for his help in the experimental setup and Mr. Dan Budescu for his excellent design and help on the prototype of the second snake robot, MS-2. The authors would also like to thank Dr. Howard Moraff for suggesting the term "Obstacle Accommodation."

\section{REFERENCES}

[1] J. S. Arora, Introduction to Optimum Design . New York: McGrawHill, 1989.

[2] R. Baldur and W. Blach, "Inflatable manipulator," SME Tech. Paper MS85-577, Robotics 9 Conf., Detroit, MI, June 1985.

[3] J. Borenstein and Y. Koren, "Real-time obstacle avoidance for fast mobile robots," IEEE Trans. Syst., Man, Cybern., vol. 19, pp. 1179-1187, Sept./Oct. 1989

[4] J. Byrd and K. R. DeVries, "A six-legged telerobot for nuclear applications development," The Int. I. Robotics Res., vol. 9, no. 2 pp. 43-52, Apr. 1990 .

[5] G. S. Chirikjian and J. W. Burdick, "Hyper-redundant robot mechanisms and their applications," IROS' 1991, Osaka, Japan.

[6] G. S. Chirikjian and J. W. Burdick, "An obstacle avoidance algorithm for hyper-redundant manipulators," in Proc. IEEE Int. Conf. Robotics Automat., Cincinnati, OH, May 13-18, 1990, pp. 625-631.
[7] W. I. Clement and R. M. Inigo, "Design of a snake-like manipulator," Robotics and Autonomous Systems, Vol. 6, pp. 265-282, April 1990.

[8] C. Gans, "How snakes move," Scientific Amer., vol. 222, pp. 82-96, 1970.

[9] S. Hirose, Biomechanical Eng. (in Japanese). Tokyo: Kogyo Chosakai, 1987.

[10] S. Hirose and A. Morishima, "Design and control of a mobile robot with an articulated body," The Int. J. Robotics Res., vol. 9, no. 2, pp. 99-114, Apr. 1990.

[11] O. Khatib, "Real-time obstacle avoidance for manipulators and mobile robots," IEEE Int. Conf. Robotics Automat., St. Louis, MO, Mar. 1985 pp. 500-505.

[12] Y. Koren, Robotics for Engineers. New York: McGraw-Hill, 1985.

[13] Y. Shan and Y. Koren, "Obstacle accommodated motion control of a plannar manipulator," in Proc. Int. Conf. Contr. Robotics, Vancouver, BC, Canada, Aug. 4-7, 1992, pp. 62-65.

[14] "Obstacle accommodation of a snake robot" to be published.

[15] Y. Shan, "Obstacle accommodation: Mechanics, control, and applications," Univ. Mich., Ann Arbor, 1992.

[16] W. K. Taylor, D. Lavie, and I. I. Esat. “A curvilinear snake arm robot with gripper-axis fibre-optic image processor feedback" Robotica vol. I, pp. 33-39, 1983.

[17] E. Verriest, "Efficient motion planning for a planar multiple link robot based on differential friction," in Amer. Contr. Conf. Proc. vol. III, 1989, pp. 2364-2365.

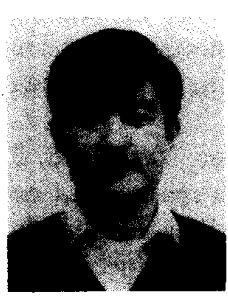

Yansong Shan received the BSME degree in 1982 from Shanghai Jiao-Tong University, Shanghai, China, the MSME degree in 1986 from the University of Tokyo, Tokyo, Japan, and the Ph.D. degree in 1992 from the University of Michigan, Ann Arbor, all in mechanical engineering.

From 1988 to 1990 , he was a Development Engineer at CardioPulmonic Inc. After receiving the Ph.D. degree, he joined the Department of Mechanical Engineering at the University of Michigan, Ann Arbor, where he is currently conducting researches on manufacturing systems, robotics, and man-machine systems.

Dr. Shan was a Rackham Predoctoral Fellow at the University of Michigan during his Ph.D. study and he is a member of Tau Beta Pi.

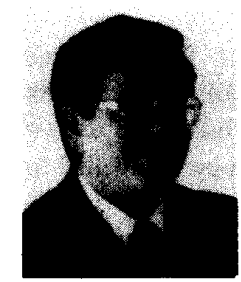

Yoram Koren (M'76-SM"88) received the B.Sc. and M.Sc. degrees in electrical engineering in 1967 and the D.Sc. degree in mechanical engineering in 1970 from the Technion, Israel Institute of Technology, Haifa, Israel.

$\mathrm{He}$ is a Professor in the Department of Mechanical Engineering at the University of Michigan, Ann Arbor. He has 25 years of research, teaching, and consulting experience in the automated manufacturing field. $\mathrm{He}$ is the author of more than 120 technical papers and three books, and the inventor of three U.S. patents in robotics. His book, Computer Control of Manufacturing Systems (McGraw Hill, 1983) is used as a textbook at major universities and received the 1984 Textbook Award from SME. His book, Robotics for Engineers, (McGraw Hill, 1985) was translated into Japanese and French and is used by engineers throughout the world.

Dr. Koren is a Fellow of ASME, an Active Member of CIRP, and a Fellow of SME Robotics- International. 